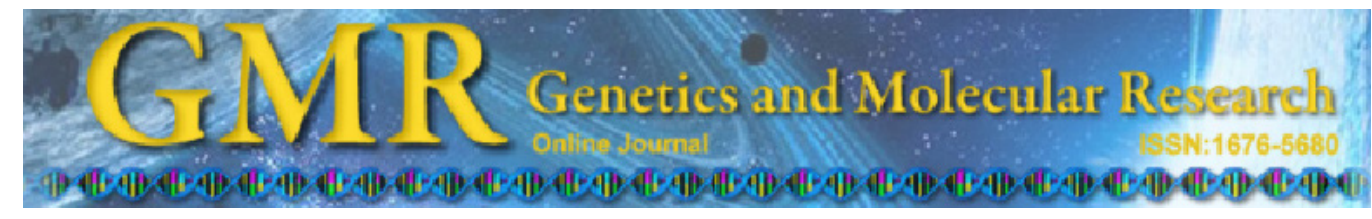

\title{
Structure and immune expression analysis of hemoglobin genes from the blood clam Tegillarca granosa
}

\author{
Y.B. Bao ${ }^{1}$, Q. Wang ${ }^{1}$, X.M. Guo ${ }^{2}$ and Z.H. Lin $^{1}$ \\ ${ }^{1}$ College of Biological and Environmental Sciences, \\ Zhejiang Wanli University, Ningbo, Zhejiang, China \\ ${ }^{2}$ Haskin Shellfish Research Laboratory, \\ Institute of Marine and Coastal Sciences, Rutgers University, \\ Port Norris, NJ, USA \\ Corresponding authors: Z.H. Lin / Y.B. Bao \\ E-mail: zhihua9988@126.com / bobbao2001@gmail.com
}

Genet. Mol. Res. 12 (3): 3110-3123 (2013)

Received May 26, 2012

Accepted November 10, 2012

Published February 28, 2013

DOI http://dx.doi.org/10.4238/2013.February.28.5

\begin{abstract}
Hemoglobin $(\mathrm{Hb})$ is the major protein component of erythrocytes in animals with red blood, although it can serve additional functions beyond the transport of oxygen. The blood clam (Tegillarca granosa) is one of the few mollusks that has $\mathrm{Hb}$, although the structure and function of molluskan Hbs remain unclear. We characterized two unique and highly compartmentalized blood clam hemoglobin genes, $T g$ - $H b I I A$ and $T g-H b I I B$, at the molecular level. The full-length cDNA of $T g$ - $H b I I A$ was $731 \mathrm{bp}$ with a 450 -bp open reading frame encoding 150 amino acids; that of $T g-H b I I B$ was $698 \mathrm{bp}$, with a 456-bp open reading frame encoding 152 amino acids. Their intronic regions were amplified by PCR. The two genes showed the typical 2 intron/3 exon organization found in T. granosa. The 3-D structures of the three blood clam Tg-Hbs were predicted using the SWISS-MODEL Protein Modeling Server, and a phylogenetic analysis was conducted to investigate its evolution. As quantified by qRT-PCR, the expression levels of $T g$ - $H b I I A$ and $T g$-HbIIB were significantly upregulated upon challenge by Vibrio
\end{abstract}


parahaemolyticus, lipopolysaccharides, and peptidoglycans. Three $\mathrm{Hb}$ isoforms, $T g-H b I, T g-H b I I A$, and $T g-H b I I B$, were found. Specific structures and evolutionary features were found in these molluskan $\mathrm{Hb}$ genes. Challenge experiments indicated that $\mathrm{Tg}-\mathrm{Hbs}$ are involved in immune defense responses against bacterial infection and bacterial pathogenic factors. As this is the first functional research on $\mathrm{Hb}$ genes in the blood clam, our findings provide new insight into the innate immune defense mechanisms of T. granosa.

Key words: Hemoglobins; Tegillarca granosa; Gene structure; Immune response; qRT-PCR

\section{INTRODUCTION}

The marine blood clam (Tegillarca granosa) is a bivalve mollusk of the family Arcidae. It is a major fishery and aquaculture species on the east coast of China and southeast Asia (Abbas Alkarkhi et al., 2008). Clam farming in Asia is experiencing serious problems of disease outbreaks and environmental pollution (Vuddhakul et al., 2006; Jung et al., 2007; Abbas Alkarkhi et al., 2008). In mollusks, hemocytes are considered to be immune cells, and studies have shown that enzymes and molecules involved in the immune defense reaction are present in hemocytes when mollusks are infected with bacteria or viruses (Bao et al., 2011a,b).

Hemoglobin is the main protein component of hemocytes. They are heme-containing proteins that bind $\mathrm{O}_{2}$ and other gaseous ligands between the iron atom at the center of the porphyrin ring and a histidine residue on the polypeptide chain (Abbas Alkarkhi et al., 2008). In addition to supporting aerobic metabolism by providing $\mathrm{O}_{2}$, hemoglobins fulfill a broad range of other functions including $\mathrm{O}_{2}$ sensing, detoxification of harmful reactive oxygen species (ROS), signaling in oxygen-dependent metabolic pathways, and production and elimination of NO (Terwilliger, 1998; Wajcman et al., 2009; Ebner et al., 2010). Hemoglobin (Hb) is also a source of antibacterial peptides in many animals; intact hemoglobin and its fractions exhibit antimicrobial activity against Gram-positive and -negative bacteria (Parish et al., 2001; Nedjar-Arroume et al., 2008). Human $\mathrm{Hb}$ can induce oxidative stress that may contribute to MMP-9 activation and BBB dysfunction, leading to apoptosis in vivo (Katsu et al., 2010).

Most mollusks and other invertebrates use hemocyanin for oxygen transport. Interestingly, Arcidae blood clams contain the red hemoglobin pigment of vertebrates. The pigment is found simply dissolved (not in corpuscles) in the blood and tissues, and it colors the muscle red. Peculiar functions have been reported in the clam Lucina pectinata, where hemoglobin I is a sulfide-reactive protein that reacts with $\mathrm{H}_{2} \mathrm{~S}$ to form ferric hemoglobin sulfide (Rivera et al., 2008).

Blood clams, such as T. granosa, Scapharca inaequivalvis, Anadara trapezia, and S. broughtonii, contain 2 types of hemoglobins that cooperatively bind oxygen (Chiancone et al., 1981; Royer et al., 1985). The major hemoglobin component, HbII, is formed by two polypeptide chains ( $\mathrm{A}$ and $\mathrm{B}$ ) that associate to form an $\mathrm{A}_{2} \mathrm{~B}_{2}$ heterotetramer. The minor hemoglobin component, $\mathrm{HbI}$, is made of a third type of chain that associates with itself to form a homodimer. These globin chains are encoded by different genes. $T$. granosa $\mathrm{HbI}(\mathrm{Tg}-\mathrm{HbI})$ was cloned and characterized in our previous study (Bao et al., 2011b).

Investigation of the hemoglobin genes could advance our understanding of the functions 
of hemocytes in the innate immune system and provide new perspective for disease control in the blood clam. The purposes of this study were to 1) clone the full-length cDNA and gDNA of $\mathrm{Hb}$ genes from $T$. granosa 2 ) compare and investigate the diversity of Tg-Hbs and evolution of molluskan $\mathrm{Hbs}$, and 3) detect mRNA temporal expression of $\mathrm{Tg}$ - $\mathrm{HbII}$ in hemocytes of $\mathrm{T}$. granosa after Vibrio parahaemolyticus, lipopolysaccharide (LPS), and peptidoglycan (PGN) stimulation.

\section{MATERIAL AND METHODS}

\section{Injection challenge, tissue collection, and cDNA synthesis}

Blood clams, averaging about $30 \mathrm{~mm}$ in shell length, were collected from a clam farm in Ningbo and acclimatized in seawater tanks $\left(10 \mathrm{~m}^{3}\right)$ for 1 week before processing. The seawater temperature was $23^{\circ} \pm 1.0^{\circ} \mathrm{C}$ and the salinity was $30 \%$ throughout the experiments. Seawater was changed every day. For challenge experiments, 60 clams were injected with $V$. parahaemolyticus (MM21). For each individual, $20 \mu \mathrm{L}$ live $V$. parahaemolyticus suspended in PBS $\left(2 \times 10^{8} \mathrm{CFU} / \mathrm{mL}, \mathrm{pH} 7.2\right)$ was injected into the foot muscle. Four individuals were randomly sampled at $1.5,3,6,12,24$, and $48 \mathrm{~h}$ after injection. Clams challenged with LPS (L6511, Sigma, USA) and PGN (53243, Sigma) were injected with $20 \mu \mathrm{L} 0.2 \mathrm{mg} / \mathrm{mL}$ of each reagent in $0.85 \% \mathrm{NaCl}$ as described for the $V$. parahaemolyticus group. Un-injected clams and 60 clams injected with $20 \mu \mathrm{L}$ PBS were used as the time 0 (at $0 \mathrm{~h}$ ) and control groups, respectively. Tissues of healthy clams, including hemocytes, adductor muscle, foot, digestive tract, gill, and mantle, were collected from 3 clams to investigate tissue-specific expression of $T g-H b I I$. Total RNA was extracted from tissues with the TRIzol reagent (Invitrogen, USA) according to manufacturer protocols. The extracted RNA was then treated with RQ1 RNase-Free DNase (Promega, USA) to remove contaminating DNA. cDNAs were synthesized from total RNA with M-MLV reverse transcriptase (Promega) and Oligo(dT) primer (Sangon, China).

\section{cDNA library construction and expressed sequence tag (EST) analysis}

A cDNA library was constructed from the hemocytes of a blood clam, using the Creator Smart cDNA Library Construction Kit (Clontech, USA) and the TRIMMER-DIRECT kit (Evrogen, Russia). Random sequencing of the library using T7 primer (Table 1) yielded 2278 successful sequencing reactions. BLAST analysis of all ESTs revealed that a 445-bp EST was highly similar to hemoglobin in Scapharca kagoshimensis (Score 208 bits, Expect 2.00E-52, Identities $75 \%$, GenBank accession No. ACN90947), another EST (386 bp) was highly similar to polymeric hemoglobin in Barbatia lima (Score 139 bits, Expect 2.00E-31, Identities 78\%, GenBank accession No. BAA09964); both ESTs were used to design primers for cloning the $\mathrm{Hb}$ genes.

\section{Cloning of the full-length cDNA of $\mathrm{Tg}-\mathrm{HbIIA}$ and $\mathrm{Tg}-\mathrm{HbIIB}$ genes}

For Tg-HbIIA cDNA cloning, gene-specific primers Tg-HbIIA-F1 and Tg-HbIIA-R1 (Table 1) were designed based on the partial sequence data of HbIIA. The 3'-RACE PCR was performed with the gene-specific $\mathrm{Tg}-\mathrm{HbI}$-F1 primer and universal vector primer T7. PCR cycling conditions included 35 cycles of $94^{\circ} \mathrm{C}$ for $40 \mathrm{~s}, 56^{\circ} \mathrm{C}$ for $40 \mathrm{~s}$, and $72^{\circ} \mathrm{C}$ for $50 \mathrm{~s}$, and an extension of $72^{\circ} \mathrm{C}$ for 10 $\mathrm{min}$. The 5'-RACE PCR was performed with the gene-specific Tg-HbIIA-R1 primer and universal 
vector primer T3. PCR cycling conditions were as described above, with a $58^{\circ} \mathrm{C}$ annealing temperature. For $T g$ - $H b I I B$ cDNA cloning, gene-specific primers $T g-H b I I B$-F1 and $T g-H b I I B$-R1 (Table 1) were designed based on the partial HbIIB sequence data. The strategy for HbIIB RACE is the same as for $H b I I A$, but with different primers and annealing temperatures. PCR products were cloned into the pMD18-T simple vector (TaKaRa, Japan) and sequenced in both directions with primers M1347 and RV-M. The sequences were verified and subjected to cluster analysis.

\begin{tabular}{|c|c|c|}
\hline Primers & Sequence (5'-3') & Application \\
\hline \multicolumn{3}{|l|}{ RACE } \\
\hline Tg-HbIIA-F1 & TTTGTTTGCCTTGAGACCAG & 3'-RACE PCR \\
\hline Tg-HbIIA-R1 & ATTTCAATCTGGATGGGTCA & 5'-RACE PCR \\
\hline Tg-HbIIB-F1 & ATTCGGAGCAATCAATGAGC & 3'-RACE PCR \\
\hline Tg-HbIIB-R1 & GTCTGGTCTCAAGGCAAACA & 5'-RACE PCR \\
\hline $\mathrm{T} 3$ & ATTAACCCTCACTAAAGGGA & Universal RACE primer \\
\hline $\mathrm{T} 7$ & TAATACGACTCACTATAGGG & Universal RACE primer \\
\hline M13-47 & CAGCACTGACCCTTTTGGGACCGC & Sequence primer \\
\hline RV-M & GAGCGGATAACAATTTCACACAGG & Sequence primer \\
\hline \multicolumn{3}{|c|}{ 67 } \\
\hline Tg-HbIIA-GSP1-1 & ACTGACAAACAATTACGACA & First outer primer for first genome walking \\
\hline Tg-HbIIA-GSP1-2 & CGTTCATTTGCTCCTAGTGACG & Second nested primer for first genome walking \\
\hline Tg-HbIIA-GSP1-3 & CATGTCCATTGATGGTTGGTCC & Third nested primer for first genome walking \\
\hline Tg-HbIIA-GSP2-1 & GTCAACGTGATTGCGTGTCCTCTC & First outer primer for second genome walking \\
\hline Tg-HbIIA-GSP2-2 & CATGGATGTTATGGTGCTGTATC & Second nested primer for second genome walking \\
\hline Tg-HbIIA-GSP2-3 & TGTTTAATCAAGCAATGTTACACCGT & Third nested primer for second genome walking \\
\hline Tg-HbIIB-intron-F & CACAGACCCAGAGGTTGTTAAAGAC & Forward primer for HbIIB intron amplifying \\
\hline Tg-HbIIB-intron-R & GCCGCTCTGATAACATCAATAAGTT & Reverse primer for $\mathrm{HbIIB}$ intron amplifying \\
\hline \multicolumn{3}{|l|}{ qRT-PCR } \\
\hline Tg-HbIIA-Real-F & GCACAGGCTTAATGCTTATGGG & qRT-PCR forward primer for Tg-HbIIA \\
\hline Tg-HbIIA-Real-R & ACATTTCAATCTGGATGGGTCA & qRT-PCR reversed primer for Tg-HbIIA \\
\hline Tg-HbIIB -Real-F & GTAGATTCAGTGCCACTTGGGTTAA & qRT-PCR forward primer for Tg-HbIIB \\
\hline Tg-HbIIB -Real-R & TGATGTCTTTAACAACCTCTGGGTC & qRT-PCR reversed primer for Tg-HbIIB \\
\hline Tg-18S rDNA-F & CTTTCAAATGTCTGCCCTATCAACT & qRT-PCR forward primer for $18 \mathrm{~S}$ rDNA \\
\hline Tg-18S rDNA-R & TCCCGTATTGTTATTTTTCGTCACT & qRT-PCR reversed primer for $18 \mathrm{~S}$ rDNA \\
\hline
\end{tabular}

\section{Nucleotide and amino acid sequence analysis}

Nucleotide and deduced amino acid sequences of $T g$ - $H b I I A$ and $T g$ - $H b I I B$ cDNA were analyzed with DNAMAN 5.2.2. Hb sequences from different organisms were aligned in NCBI BLAST. A multiple sequence alignment was created with ClustalW (http://www.ebi.ac.uk/ clustalw/), and a neighbor-joining (NJ) phylogenetic tree of $\mathrm{Hb}$ was drawn in MEGA 3.1 (http:// www.megasoftware.net). The calculated molecular mass and theoretical isoelectric points were predicted by the EMBOSS model of protein isoelectric point (http://isoelectric.ovh.org/).

\section{Cloning and analysis of genomic $\mathrm{Tg}-\mathrm{HbIIA}$ and $\mathrm{Tg}-\mathrm{HbIIB}$}

Genomic DNA was purified from the adductor muscle with the Genomic DNA Purification Kit (Promega). Genomic Tg-HbIIA sequence was obtained through a genome walking approach with the Genome Walking Kit (TaKaRa). Three gene-specific primers (Table 1) based on known sequences of the 3'-flanking region of $T g$-HbIIA and shorter arbitrary degenerates (AP1, AP2, AP3, and AP4) were used for every genome walk. All gene-specific primers used for amplifying the introns and the promoter region are listed in Table 1. The sequences 
of the 4 short degenerate AP primers from the kit are unknown (proprietary). Reaction volume and tertiary amplification cycling conditions were according to the manufacturer protocol. Specific PCR products were cloned and sequenced. Genomic sequences of $T g-H b I I B$ were obtained using 2 pairs of primers designed for the 5 '- and 3 '-ends of the cDNA sequence (Table 1). Intron location and gene structure for both genes were confirmed by comparison with the cDNA sequences of $T g$-HbIIA and $T g$-HbIIB and the $S$. inaequivalvis HbII genes.

\section{Multiple sequence alignment, tertiary structure prediction, and phylogenetic analysis of $\mathbf{T g}-\mathrm{HbII}$}

The presumed tertiary structure was established using the SWISS-MODEL prediction algorithm (http://swissmodel.expasy.org/) and displayed in Deep View/Swiss-Pdb Viewer version 3.7. An unrooted phylogenetic tree of molluskan Hbs was constructed based on amino sequence alignment by the NJ algorithm embedded in MEGA 3.1. Branching reliability was tested by bootstrap re-sampling (1000 pseudo-replicates).

\section{Quantitative real-time PCR of $\mathbf{T g}-\mathrm{HbII}$ genes}

Expression of $T g$ - $H b I I A$ and $T g$ - $H b I I B$ transcripts in tissues and the temporal expression profile in hemocytes after $V$. parahaemolyticus, LPS, and PGN challenge were measured with the SYBR Green quantitative real-time Stratagene Mx3000P Real-Time PCR System. $T g$-HbIIA-specific primers $T g$-HbIIA-Real-F and Tg-HbIIA-Real-R (Table 1) were used to amplify a 185 -bp product, which was sequenced to verify PCR specificity. $T g$ - $H b I I B$-specific primers $T g$-HbIIB-Real-F and $T g-H b I I B$-Real-R (Table 1 ) were used to amplify a 97-bp product for real-time PCR. Clam 18S rDNA primers Tg-18S rDNA-F and Tg-18S rDNA-R (Table 1) were used to amplify a 195-bp fragment as a control to verify the reverse transcription and calibrate the cDNA template. On a 96-well plate, each sample was run in triplicate along with the internal control. The reaction components, thermal profile, and data analysis were conducted as previously described (Bao et al., 2011b). All data are provided in terms of relative mRNA and reported as means \pm standard error $(\mathrm{N}=4)$. The data were then subjected to the least significant difference test to identify differences in mean values between treatments. A $\mathrm{P} \leq 0.05$ was considered to be significant. Statistical analysis was performed with SPSS 13.

\section{RESULTS}

\section{cDNA library construction and EST analysis}

A normalized full-length cDNA library was constructed from blood clam hemocytes. From this library, 2278 ESTs from the 5'-end of cDNA clones with an average length of $459 \mathrm{bp}$ were obtained. Cluster analysis identified 1501 unique sequences containing 173 contigs and 1328 singletons. Two ESTs were highly homologous to HbII from S. kagoshimensis and B. lima.

\section{cDNA cloning and sequence analysis of $\mathrm{Tg}-\mathrm{HbII}$}

A 731-bp nucleotide sequence representing the complete cDNA sequence of $\mathrm{Tg}$ - $\mathrm{HbIIA}$ 
and 698 bp of $T g-H b I I B$ was obtained by assembling corresponding ESTs amplified with specific primers. The sequences were deposited in GenBank under accession No. HQ729976 for $T g$-HbIIA and HQ149306 for Tg-HbIIB. The complete sequence of $T g$-HbIIA cDNA contained a 5'-untranslated region (UTR) of $63 \mathrm{bp}$, a 3'-UTR of $218 \mathrm{bp}$ with a poly(A) tail, and an open reading frame (ORF) of $450 \mathrm{bp}$ encoding a polypeptide of 150 amino acids. The molecular mass calculated of the deduced mature $T g$-HbIIA protein was $16.23 \mathrm{kDa}$, and the theoretical isoelectric point was 8.84. The nucleotide and deduced amino acid sequences of $T g-H b I I A$ are shown in Figure 1A. The complete sequence of $T g-H b I I B$ cDNA contained a 5'-UTR of $61 \mathrm{bp}$, a 3'-UTR of $181 \mathrm{bp}$ with a poly(A) tail, and an ORF of $456 \mathrm{bp}$ encoding a polypeptide of 152 amino acids. The molecular mass calculated of the deduced mature $T g-H b I I B$ protein was $17.23 \mathrm{kDa}$, and the theoretical isoelectric point was 5.74. The nucleotide and deduced amino acid sequences of $T g$ HbIIB are shown in Figure 1B. Heme-binding sites are also shown in Figure 1.

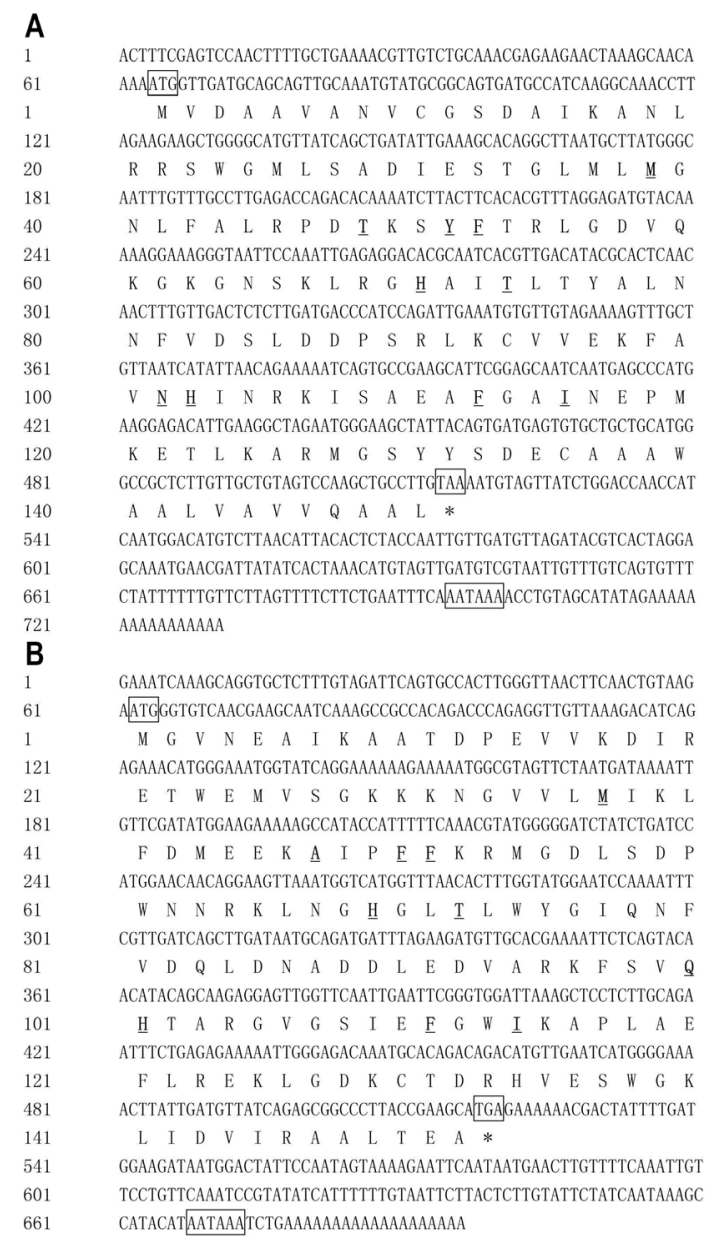

Figure 1. Nucleotide and deduced amino acid sequences of Tg-HbIIA (A) and Tg-HbIIB (B). Letters in boxes are the start codons (ATG), the stop codons (TAA or TGA), and the polyadenylation signal sequences (ATTAAA). The asterisk indicates the stop codon. The heme binding sites are in bold and underlined. 


\section{Homologous and phylogenetic analysis of $\mathrm{Tg}-\mathrm{HbII}$}

The $\mathrm{Hb}$ amino acid sequence alignment showed $32 \%$ identity and $47.7 \%$ similarity between $\mathrm{Tg}$ - $\mathrm{HbIIA}$ and $\mathrm{Tg}-\mathrm{HbIIB}, 56 \%$ identity and $68.7 \%$ similarity between $\mathrm{Tg}-\mathrm{HbI}$ (HQ149305) and $\mathrm{Tg}-\mathrm{HbIIA}$, and $38.6 \%$ identity and 53.6\% similarity between $\mathrm{Tg}-\mathrm{HbI}$ and $\mathrm{Tg}$ HbIIB (Figure 2A). HbIIA was highly conserved among 3 species of blood clams: T. granosa, $S$. inaequivalvis, and S. kagoshimensis (Figure 2B); however, HbIIB showed relatively little conservation between T. granosa and Scapharca (Figure 2C). Alignment with HbII from other mollusks (A. trapezia, B. lima) only showed conserved structural motifs, functional domain, heme-binding sites, etc. (Figure 2C). The tertiary structures of $3 \mathrm{Tg}$-Hbs were predicted by the SWISS-MODEL prediction algorithm (Figure 3). The typical structure of $\mathrm{Hb}$ is composed of a heme prosthetic group held within the alpha helical globular fold of a 100- to 200-amino acid protein monomer by a covalent bond to a histidine side chain termed the "proximal histidine". Overall, the 3-D structures of the Tg-Hbs are similar, except for a divergence in the number and length of the alpha helices. The amino acids forming the heme pocket are more well conserved than other regions. Only one residue divergence exists between $\mathrm{Tg}-\mathrm{HbI}$ and $\mathrm{Tg}$ - $\mathrm{HbIIA}$, i.e., the addition of VAL ${ }^{143}$ in $\mathrm{Tg}-\mathrm{HbI}$. In comparison to $\mathrm{Tg}-\mathrm{HbI}$ and $\mathrm{Tg}$ - $\mathrm{HbIIA}, \mathrm{Tg}-\mathrm{HbIIB}$ was relatively more different; residues $\mathrm{ALA}^{47}, \mathrm{PHE}^{50}, \mathrm{GLN}^{100}$, and ILE ${ }^{145}$ were unique in $\mathrm{Tg}$ - $\mathrm{HbIIB}$.

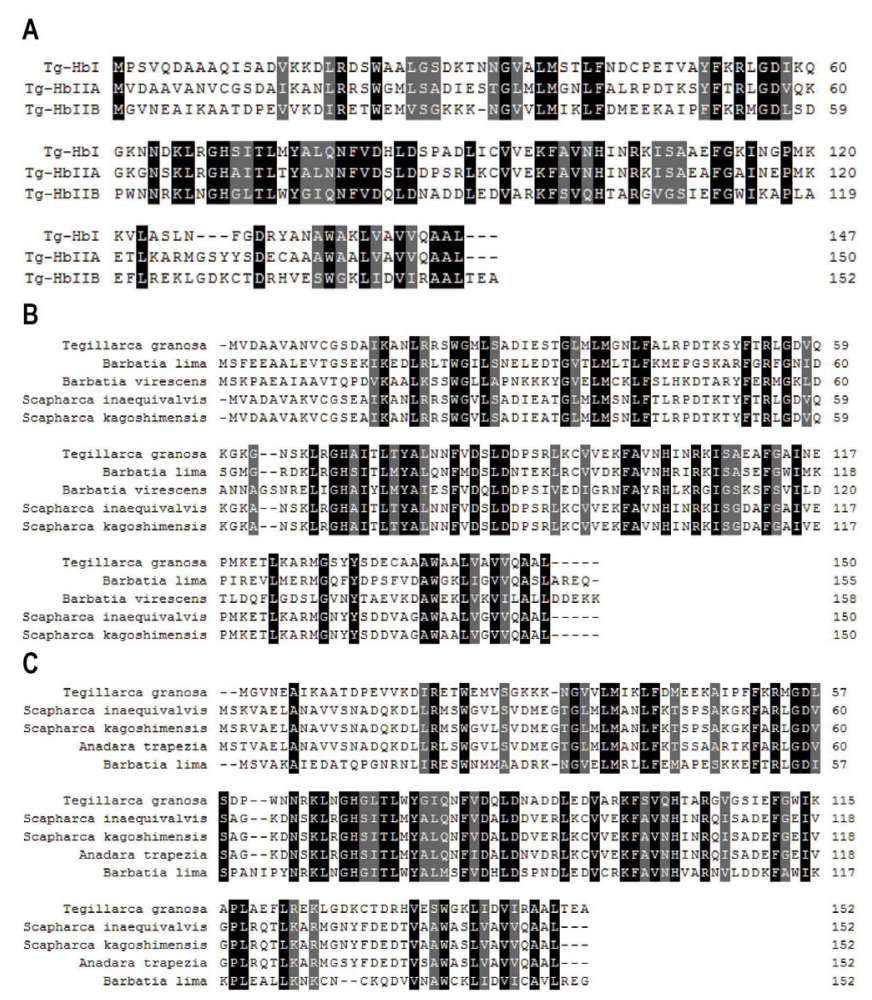

Figure 2. A. Alignment of deduced amino acid sequences of $3 \mathrm{Tg}-\mathrm{Hbs}$ from the blood clam Tegillarca granosa. B. Alignment of HbIIA from 5 clams. C. Alignment of HbIIB from 5 clams. Conserved amino acid residues are shaded in black, and similar amino acids are shaded in gray. 


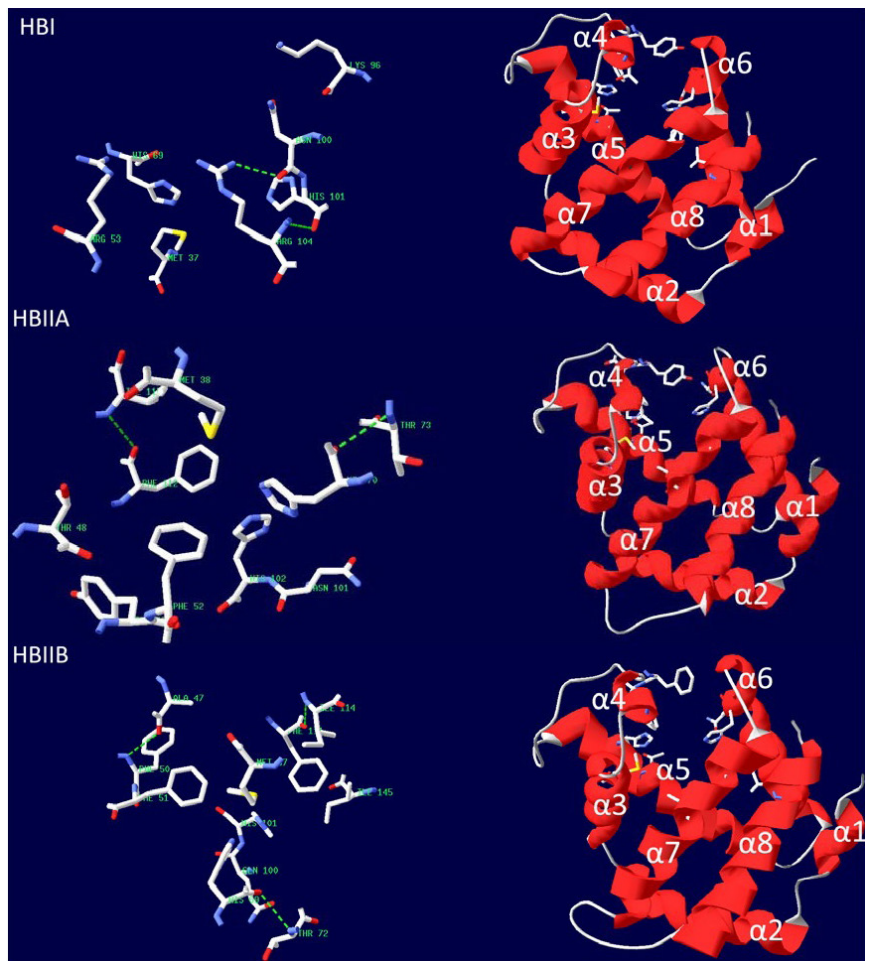

Figure 3. Predicted 3-D spatial structures of Tg-HbI, Tg-HbIIA, and Tg-HbIIB using the SWISS-MODEL program and displayed with the Swiss-PDB viewer. The $\alpha$-helices are shown as red ribbons and the amino acids essential for heme binding are shown in detail with the amino acid skeleton and labeling.

An NJ tree constructed on the basis of 25 molluskan $\mathrm{Hb}$ sequences is shown in Figure 4. Two major monophyletic groups, one including the deep sea clam genus Calyptogena, tropical clam genus Lucina and Phacoides, and the other including other clams, are included. The Antarctic clam Yoldia eightsi does not cluster with either group. The Hb-based tree provides information on the evolution of the species and genes.

\section{Genomic structure of $\mathrm{Tg}$ - $\mathrm{HbII}$ genes}

Figure 5 shows the genomic organization and structural comparison of $\mathrm{Tg}-\mathrm{HbI}$ (GenBank accession No. JQ713118) and 2 HbII genes in the blood clams T. granosa and S. inaequivalvis. The full-length $\mathrm{Tg}$-HbIIA genomic DNA consisted of about $1797 \mathrm{bp}$ (JQ713119) and contained 3 exons and 2 introns. The $T g-H b I I B$ genomic DNA consisted of about $3203 \mathrm{bp}$ (JQ713120) and contained 3 exons and 2 introns. All introns were located within the ORF. All exon-intron junctions followed the consensus rule of the splice acceptor-AG/GT-splice donor for splicing. The cDNA length of $2 \mathrm{Tg}$-HbII genes was similar in length; however, the length of the $T g-H b I I B$ gDNA was much longer than that of $T g-H b I I A$ due to the long first intron (2179 bp) of Tg-HbIIB. Compared with S. inaequivalvis HbII genes (Si-HbII), the HbIIA gene 


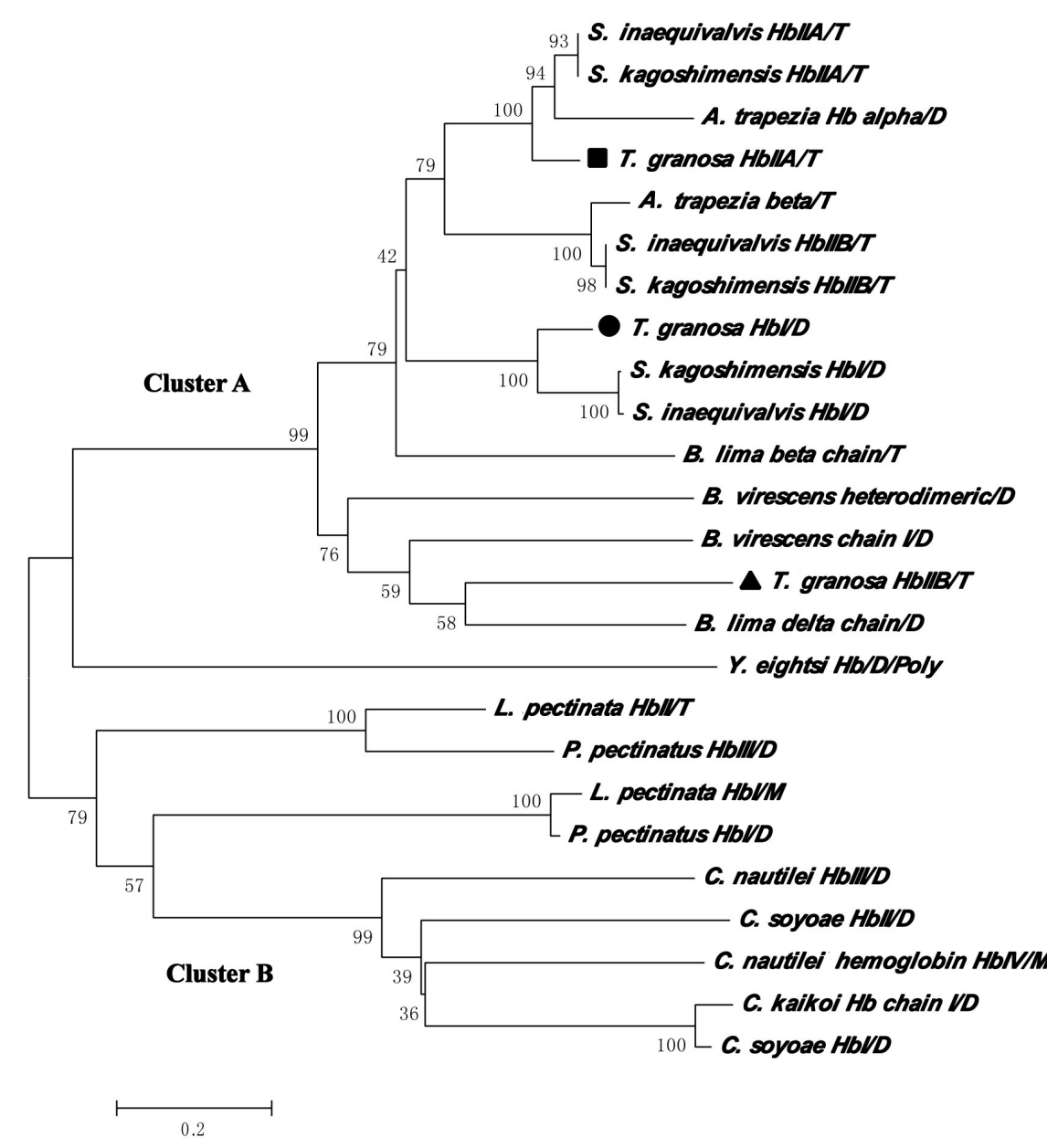

Figure 4. Phylogenetic tree for molluskan hemoglobins, constructed with the neighbor-joining method. The numbers at the forks are bootstrap values. The species and the GenBank accession Nos. are as follows: Scapharca inaequivalvis (P14821, AAB51308, CAA50510), S. kagoshimensis (ACN90947, ACN90948, ACN90946), Anadara trapezia (P04251, P14394), Tegillarca granosa (HQ729976, HQ149306, HQ149305), Barbatia lima (BAA09965, BAA09967), B. virescens (BAA09587, AAB24577), Yoldia eightsi (AAM00251), Lucina pectinata (P41261, P41260), Phacoides pectinatus (P41262, P41260), Calyptogena nautilei BAD34605, BAD34606), C. soyoae (P14805, P14528), C. kaikoi (BAD34601). Protein structures are indicated as monomeric (M), dimeric (D), or tetrameric $(\mathrm{T})$.

structure was more conserved than that of HbIIB; the 3 exons are of the same length in HbIIA (Figure 5). The $T g-H b I I B$ gDNA sequence was longer than that of Si-HbIIB. The length of exon 2 was conserved ( 220 bp) in all blood clam hemoglobins. Further, many minisatellite tandem sequences were found in the $\mathrm{Hb}$ introns, including 2 dimers in $T g-H b I, 2$ dimers and 1 tetramer in $T g-H b I I A, 5$ dimers and 3 trimers in $T g$ - $H b I I B$. Overall, the gene structures of $T g$ $\mathrm{HbI}$ and $\mathrm{Tg}$ - $\mathrm{HbIIA}$ were more similar than $\mathrm{Tg}$ - $\mathrm{HbIIA}$ and $\mathrm{Tg}-\mathrm{HbIIB}$, although $\mathrm{Tg}$ - $\mathrm{HbIIA}$ and $\mathrm{Tg}$-HbIIB associate to form heterologous tetramers. 


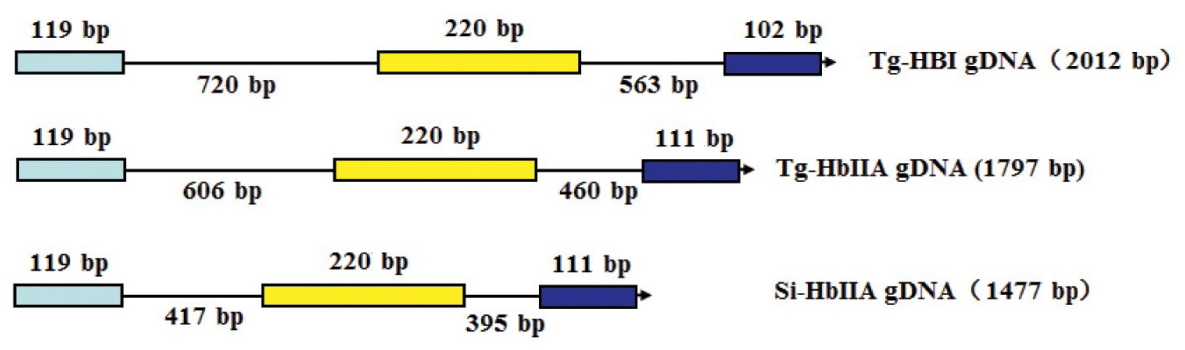

Tg-HbIIB gDNA (3203 bp)

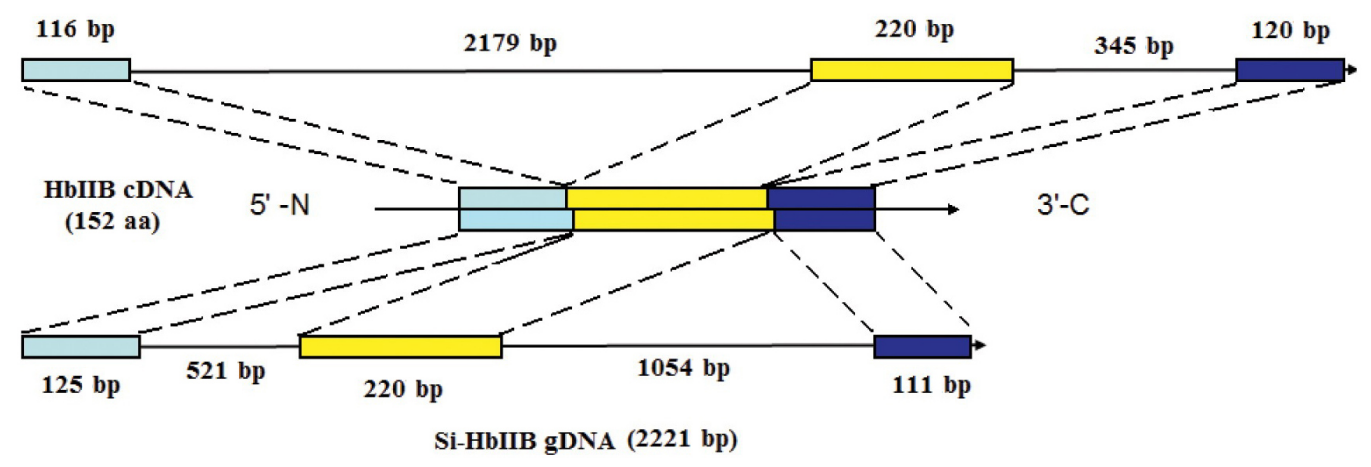

Figure 5. Genomic organization and comparison of Tg-HbI and two kinds of HbII gene structure between the blood clam Tegillarca granosa and Scapharca inaequivalvis. Exons 1-3 are indicated by boxes with different colors. Introns are indicated by lines and are numbered. The numbers above the boxes and below the lines are length in base pairs.

\section{Temporal expression of $\mathrm{Tg}-\mathrm{HbII}$ after challenge}

Expression of $T g-H b I I$ mRNA in clam hemocytes after $V$. parahaemolyticus, LPS, and PGN challenge was quantified by qRT-PCR, using $18 \mathrm{~S}$ rRNA as an internal control. The expression of $T g$ - $H b I I A$ and $T g$ - $H b I I B$ transcript was generally upregulated after all of these challenges, although expression levels differed (Figure 6). The quantity of $\mathrm{Tg}$ - $\mathrm{HbIIA}$ transcripts increased gradually from 1.5 to $12 \mathrm{~h}$, reaching a peak about 600 -fold greater $(\mathrm{P}<0.01)$ than at time 0 in the $V$. parahaemolyticus group, about 250 -fold in the LPS group, and about 500 -fold in the PGN group, then rapidly decreased from 12 to $24 \mathrm{~h}$ to $10-30$-fold at $48 \mathrm{~h}$ (Figure 6A). Transcripts of $T g-H b I I B$ were detected at various levels in the 3 challenge groups. In the Vibrio challenge group, the expression of $T g-H b I I B$ mRNA was similar to that of $T g$ $H b I I A$, although transcript quantities were significant lower than those of $T g$ - $H b I I A$, with the peak at $12 \mathrm{~h}$ of about 80 -fold $(\mathrm{P}<0.01)$ over time 0 . In the LPS and PGN challenge groups, the quantity of $T g$ - $H b I I B$ transcripts increased gradually from 0 to $6 \mathrm{~h}$, reaching their highest level at $6 \mathrm{~h}$ (about 20 -fold compared with time $0, \mathrm{P}<0.05$ ), and then decreasing gradually from 12 to $48 \mathrm{~h}$ (Figure 6B). In the control group (PBS), levels increased only slightly between 3 and $24 \mathrm{~h}$ due to the injection wound (less than 10-fold). No mortality occurred in the challenged and control groups. 
A

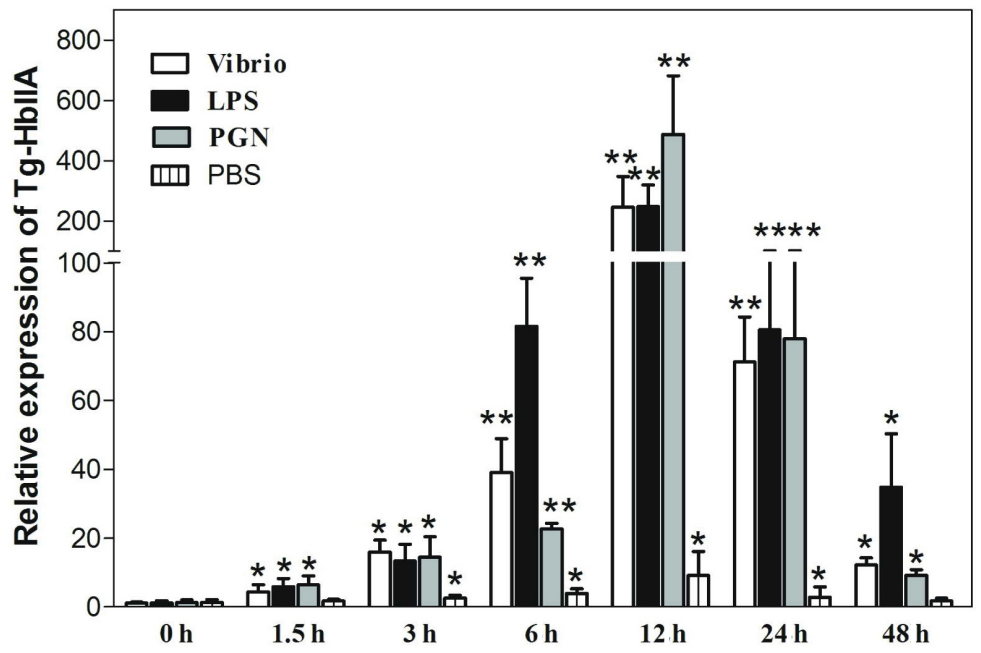

B

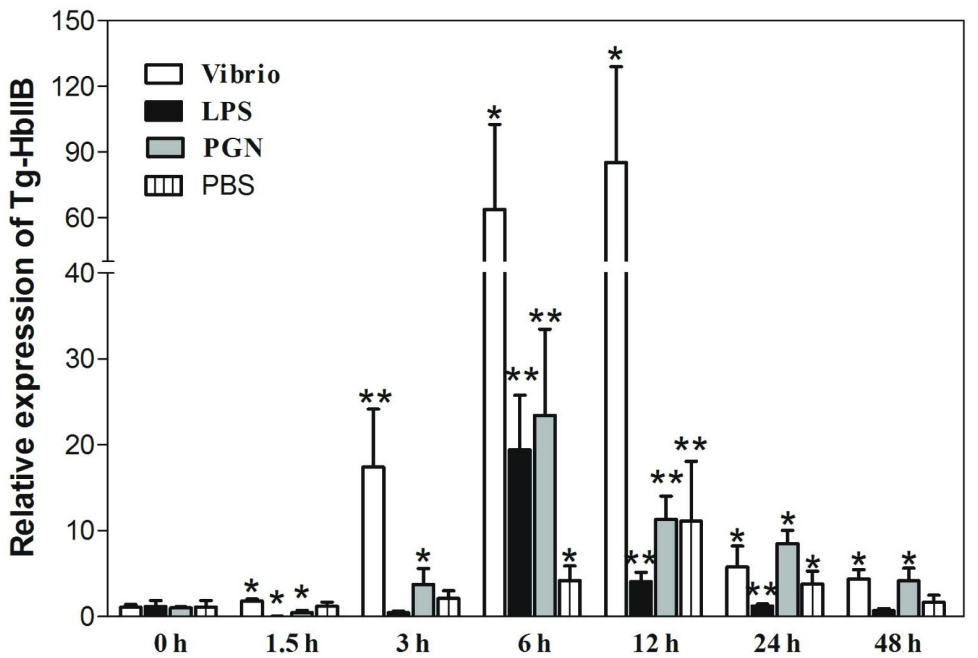

Figure 6. Real-time PCR analysis of Tg-HbIIA (A) and Tg-HbIIB (B) gene expression in hemocytes of the blood clam challenged with Vibrio parahaemolyticus, lipopolysaccharide (LPS), and peptidoglycan (PGN). Comparison of the level of Tg-HbII mRNA (relative to $18 \mathrm{~S}$ rDNA) at different time points was performed using the Student $t$-test. Data were analyzed from 4 individuals. Vertical bars are reported as means \pm standard error $(\mathrm{N}=4)$. Significant differences between challenged and zero time point are indicated with an asterisk for $\mathrm{P}<0.05$, and with two asterisks for $\mathrm{P}<0.01$.

\section{DISCUSSION}

Two components of $T g$ - $H b I I$ ( $T g$ - $H b I I A$ and $T g$ - $H b I I B)$ were cloned and compared to $\mathrm{Tg}-\mathrm{HbI}$ reported in our previous study (Bao et al., 2011a). Sequence analysis indicated that the 
$3 \mathrm{Hb}$ genes had conserved sequences in the heme-binding region, but low amino acid similarity in other regions. $\mathrm{HbI}$ and $\mathrm{Tg}$ - $H b I I A$ were more similar in terms of the primary protein or gene structure, and $T g-H b I I B$ was more divergent. This diversity probably reflects erratic evolutionary changes in $T g-H b I I B$, suggesting that they evolved differently from the ancestral $\mathrm{Hb}$ gene. Despite the relatively high cDNA and protein sequence diversity, a comparison of the 3-D structures of Tg-Hbs revealed they were highly similar, only varying in the number of amino acids in several alpha helices. In most cases, divergences in sequence and structure are parallel, but structural changes are more conserved than sequence changes in the $\mathrm{Hb}$ of T. granosa, especially in the heme pocket. The similarity in folding pattern likely stabilizes $\mathrm{Hb}$ structure and function in evolutionary terms. These results were consistent with previous reports (Lecomte et al., 2005).

Two distinct clades were observed on the evolutionary tree. Three $\mathrm{Tg}$-Hbs appeared in Cluster A, which belong to blood or ark clams. Hbs in Cluster B are clams from extreme environments, such as the deep sea and Polar Regions. In this context, the Hbs are good models for gene family evolution. The evolutionary rate of Calyptogena and Phacoides hemoglobins is faster than those of other clams, suggesting that the mutation rate might be accelerated in deep-sea animals around areas of cold seeps or hydrothermal vents (Suzuki et al., 2000; TorresMercado et al., 2003). The tropical clam Lucina is not a deep-sea clam, but its HbI is sulfidereactive and supposed to play an important role in transporting sulfide to a bacterial symbiont (Suzuki et al., 2000). The Antarctic clam Y. eightsi $\mathrm{Hb}$ gene is separated from other molluskan $\mathrm{Hb}$ genes, and this diversity may be an important part of adaptation to its polar low-temperature environment (DeWilde et al., 2003). This remarkable diversity in hemoglobin constituent chains and subunit structure suggest that gene duplication and fusion have occurred frequently during the course of the evolution of clam hemoglobins, indicating that the living environment can influence $\mathrm{Hb}$ evolution, leading to diversity of structure and function.

Vertebrate and many invertebrate globin genes have a 3 exon/2 intron organization, and the blood clam is no exception. Hinds and Blake (1985) argued that DNA is organized so that coding sequences (exons) correspond to structural as well as functional units of proteins (Go, 1981). In our study, exon 2 was conserved in $\mathrm{Hb}$ evolution in the blood clam. The central coding sequence corresponds to the heme interactive and binding functions. Minigenes that can be translated to coding sequences of $\mathrm{Hb}$ were found in blood clam S. inaequivalvis $\mathrm{HbII}$ genes (Gambacurta et al., 1998), but were not found in T. granosa or other organisms. However, many minisatellite tandem sequences were found in the introns of $\mathrm{Tg}-\mathrm{Hbs}$, especially in $T g-H b I I B$. The genomic mechanism for generation of these minisatellites remains unclear. These minisatellite repeats might be involved in transcriptional regulation of the $\mathrm{Hb}$ genes. Furthermore, polymorphisms in introns could serve as genetic markers for studying genotypephenotype associations and marker-assisted selection.

While hemoglobin is one of the most well-characterized proteins due to its function in oxygen transport, few additional properties have been described. Recently, other functions of $\mathrm{Hb}$ have been discovered and investigated (Rizzi et al., 1994; Wittenberg and Stein, 1995; Terwilliger, 1998; Coletta et al., 1999; Weber and Vinogradov, 2001; Kawano et al., 2002; Wajcman and Kiger, 2002; Wajcman et al., 2009). ROS-mediated antimicrobial activity is a powerful host defense mechanism. Studies have shown that the respiratory protein hemoglobin can produce ROS (Jiang et al., 2007). Recently, studies have also shown that antibacterial peptides derived from hemoglobins and intact hemoglobins have antibacterial activity in 
some higher vertebrates (Parish et al., 2001; Nedjar-Arroume et al., 2008). Studies have also demonstrated that hemoglobin is able to detect, scavenge, and detoxify NO; NO detoxification has been linked to bacterial resistance (Gardner and Gardner, 2002; Wajcman et al., 2009). Given that hemoglobin is involved in many aspects of antibacterial immunity, investigating whether hemoglobin mRNA expression responds to an immune challenge may provide preliminary evidence for an immunomodulatory role of $\mathrm{Hbs}$. In this study, the Tg-Hb mRNA transcripts in hemocytes increased markedly after $V$. anguillarum, LPS, and PGN challenge, indicating that the expression of $\mathrm{Tg}-\mathrm{Hbs}$ is significantly induced by bacterial stimulation. Studies have shown 3 ways in which $\mathrm{Hb}$ is involved in antibacterial immunity: peptides degraded from $\mathrm{Hb}, \mathrm{ROS}$ produced by $\mathrm{Hb}$ killing bacteria, and NO-mediated killing. In each case, $\mathrm{Hb}$ mRNA increases to synthesize more $\mathrm{Hb}$ protein. Our study shows that $\mathrm{Tg}-\mathrm{Hb}$ is involved in the antibacterial immune response.

\section{CONCLUSION}

The study of the structure and function of molluskan Hbs is still in its infancy. In this and the other studies cited, full-length cDNAs of $3 \mathrm{Tg}$-Hbs ( $\mathrm{Tg}-\mathrm{HbI}, \mathrm{Tg}-\mathrm{HbIIA}, \mathrm{Tg}-\mathrm{HbIIB})$ were cloned from the blood clam T. granosa. Sequence and structural analysis revealed evolutionary changes that may be significant in molluskan diversification and adaptation to different environments. $\mathrm{Tg}-\mathrm{Hb}$ mRNA expression in hemocytes was markedly upregulated after V. parahaemolyticus, LPS, or PGN challenges. These results indicate that Tg-Hbs modulate immune responses in the blood clam and provide molecular evidence for Hbs as immunomodulatory proteins in the antibacterial immune response. In future studies, we will examine the mechanism(s) of transcriptional control of $\mathrm{Tg}-\mathrm{Hbs}$ and the possible association of single nucleotide polymorphisms with the clam's resistance/susceptibility to bacterial infections.

\section{ACKNOWLEDGMENTS}

Thanks to Yinghui Dong and Lihui Huo for experiment suggestions and sample collection. Research supported by the National Science Foundation of China (\#31001097), the National High-Tech R\&D Program of China (\#2012AA10A410), the Chinese Agriculture Research System (\#CARS-48), and the Ningbo Science and Technology International Cooperation Research Projects (\#2010D10017).

\section{REFERENCES}

Abbas Alkarkhi FM, Ismail N and Easa AM (2008). Assessment of arsenic and heavy metal contents in cockles (Anadara granosa) using multivariate statistical techniques. J. Hazard. Mater. 150: 783-789.

Bao Y, Wang Q and Lin Z (2011a). Hemoglobin of the bloody clam Tegillarca granosa (Tg-HbI) is involved in the immune response against bacterial infection. Fish. Shellfish. Immunol. 31: 517-523.

Bao Y, Wang Q, Liu H and Lin Z (2011b). A small HSP gene of bloody clam (Tegillarca granosa) involved in the immune response against Vibrio parahaemolyticus and lipopolysaccharide. Fish. Shellfish. Immunol. 30: 729-733.

Chiancone E, Vecchini P, Verzili D, Ascoli F, et al. (1981). Dimeric and tetrameric hemoglobins from the mollusc Scapharca inaequivalvis. Structural and functional properties. J. Mol. Biol. 152: 577-592.

Coletta M, Gambacurta A, Clementi ME, Erba F, et al. (1999). Functional modulation of the Thr72 $\rightarrow$ Ile mutant from Scapharca inaequivalvis homodimeric hemoglobin. J. Biol. Inorg. Chem. 4: 678-683.

DeWilde S, Angelini E, Kiger L, Marden MC, et al. (2003). Structure and function of the globin and globin gene from the Antarctic mollusc Yoldia eightsi. Biochem. J. 370: 245-253. 
Ebner B, Panopoulou G, Vinogradov SN, Kiger L, et al. (2010). The globin gene family of the cephalochordate amphioxus: implications for chordate globin evolution. BMC Evol. Biol. 10: 370.

Gambacurta A, Piro MC and Ascoli F (1998). Scapharca inaequivalvis tetrameric hemoglobin A and B genes: evidence for a minigene. J. Mol. Evol. 47: 167-171.

Gardner AM and Gardner PR (2002). Flavohemoglobin detoxifies nitric oxide in aerobic, but not anaerobic, Escherichia coli. Evidence for a novel inducible anaerobic nitric oxide-scavenging activity. J. Biol. Chem. 277: 8166-8171.

Go M (1981). Correlation of DNA exonic regions with protein structural units in haemoglobin. Nature 291: 90-92.

Hinds PW and Blake RD (1985). Delineation of coding areas in DNA sequences through assignment of codon probabilities. J. Biomol. Struct. Dyn. 3: 543-549.

Jiang N, Tan NS, Ho B and Ding JL (2007). Respiratory protein-generated reactive oxygen species as an antimicrobial strategy. Nat. Immunol. 8: 1114-1122.

Jung WK, Jo HY, Qian ZJ, Jeong YJ, et al. (2007). A novel anticoagulant protein with high affinity to blood coagulation factor Va from Tegillarca granosa. J. Biochem. Mol. Biol. 40: 832-838.

Katsu M, Niizuma K, Yoshioka H, Okami N, et al. (2010). Hemoglobin-induced oxidative stress contributes to matrix metalloproteinase activation and blood-brain barrier dysfunction in vivo. J. Cereb. Blood Flow Metab. 30: 1939-1950.

Kawano T, Pinontoan R, Hosoya H and Muto S (2002). Monoamine-dependent production of reactive oxygen species catalyzed by pseudoperoxidase activity of human hemoglobin. Biosci. Biotechnol. Biochem. 66: 1224-1232.

Lecomte JT, Vuletich DA and Lesk AM (2005). Structural divergence and distant relationships in proteins: evolution of the globins. Curr. Opin. Struct. Biol. 15: 290-301.

Nedjar-Arroume N, Dubois-Delval V, Adje EY, Traisnel J, et al. (2008). Bovine hemoglobin: an attractive source of antibacterial peptides. Peptides 29: 969-977.

Parish CA, Jiang H, Tokiwa Y, Berova N, et al. (2001). Broad-spectrum antimicrobial activity of hemoglobin. Bioorg. Med. Chem. 9: 377-382.

Rivera L, Lopez-Garriga J and Cadilla CL (2008). Characterization of the full length mRNA coding for Lucina pectinata HbIII revealed an alternative polyadenylation site. Gene 410: 122-128.

Rizzi M, Wittenberg JB, Coda A, Fasano M, et al. (1994). Structure of the sulfide-reactive hemoglobin from the clam Lucina pectinata. Crystallographic analysis at 1.5 Å resolution. J. Mol. Biol. 244: 86-99.

Royer WE, Jr., Love WE and Fenderson FF (1985). Cooperative dimeric and tetrameric clam haemoglobins are novel assemblages of myoglobin folds. Nature 316: 277-280.

Suzuki T, Kawamichi H, Ohtsuki R, Iwai M, et al. (2000). Isolation and cDNA-derived amino acid sequences of hemoglobin and myoglobin from the deep-sea clam Calyptogena kaikoi. Biochim. Biophys. Acta 1478: 152-158.

Terwilliger NB (1998). Functional adaptations of oxygen-transport proteins. J. Exp. Biol. 201: 1085-1098.

Torres-Mercado E, Renta JY, Rodriguez Y, Lopez-Garriga J, et al. (2003). The cDNA-derived amino acid sequence of hemoglobin II from Lucina pectinata. J. Protein Chem. 22: 683-690.

Vuddhakul V, Soboon S, Sunghiran W, Kaewpiboon S, et al. (2006). Distribution of virulent and pandemic strains of Vibrio parahaemolyticus in three molluscan shellfish species (Meretrix meretrix, Perna viridis, and Anadara granosa) and their association with foodborne disease in southern Thailand. J. Food Prot. 69: 2615-2620.

Wajcman H and Kiger L (2002). Hemoglobin, from microorganisms to man: a single structural motif, multiple functions. C R. Biol. 325: 1159-1174.

Wajcman H, Kiger L and Marden MC (2009). Structure and function evolution in the superfamily of globins. C R. Biol. 332: 273-282.

Weber RE and Vinogradov SN (2001). Nonvertebrate hemoglobins: functions and molecular adaptations. Physiol. Rev. 81: 569-628.

Wittenberg JB and Stein JL (1995). Hemoglobin in the symbiont-harboring gill of the marine gastropod Alviniconcha hessleri. Biol. Bull. 188: 5-7. 